\title{
Near Miss Materno: Revisão da Literatura
}

\author{
Maternal Near Miss: Literature Review
}

Near Miss Materno: Revisión de la Literatura

\author{
Rodrigo da Silva Maia ${ }^{1}$ \\ Tereza Cristina Santos de Araújo ${ }^{2}$ \\ Brenda de Andrade Rodrigues ${ }^{3}$ \\ Eulália Maria Chaves Maia ${ }^{4}$
}

\section{Resumo}

Objetivo: $\mathrm{O}$ estudo objetivou caracterizar os artigos científicos sobreo near miss materno. Método: Trata-se de uma revisão narrativa, do tipo integrativa, com recorte em estudos brasileiros. A seleção de artigos fora realizada em dezembro de 2016. Resultados: Ao todo foram encontrados 33 artigos. Quando verificados os critérios de seleção permaneceram sete publicações. Os estudos revisados tratam o tema a partir de diferentes perspectivas, com predominância de pesquisas

epidemiológicas.

Complicações hipertensivas aparecem como o principal causador do fenômeno. Conclusão: Este se trata de um problema de saúde pública, que impacta à saúde materna e à saúde do bebê, sobre o vinculo e no entorno psicossocial de quem por esta é acometida.

Descritores: Morbidade; Saúde da mulher; Revisão.

\section{Abstract}

${ }^{1}$ Psicólogo. Mestre em Psicologia pela Universidade Federal do Rio Grande do Norte (UFRN). Docente da Centro Universitário Facex (UNIFACEX). Autora correspondente: : Rua Itamarati de Minas, 2982, Apto 202, Neopólis. CEP: 59088-120. Natal-RN, Brasil. E-mail: rodrigo_maia89@yahoo.com.br ${ }^{2}$ Graduanda em Psicologia pelo UNIFACEX.

${ }^{3}$ Graduanda em Fisioterapia pela UFRN.

${ }^{4}$ Psicóloga. Doutora em Psicologia Clínica pela Universidade de São Paulo (USP). Professora Titular do Departamento de Psicologia e Orientadora de Mestrado e Doutorado nos Programas de Pós-graduação em Ciências da Saúde (PPGCSA) e Pós-graduação em Psicologia (PPGPSI) da UFRN.

Recebido: Jun/2017 - Aceito: Ago/2017. 
Objective: The study aimed to characterize the scientific articles on maternal near miss. Method: This is a narrative review, of the integrative type, with a cut in Brazilian studies. The selection of articles was made in December 2016. Results: Altogether 33 articles were found. When checked the selection criteria remained seven publications. The reviewed studies treat the theme from different perspectives, with predominance of epidemiological research. Hypertensive complications appear as the main cause of the phenomenon. Conclusion: This is a public health problem, which impacts on maternal health and the health of the baby, on the link and on the psychosocial environment of those who are affected by it.

Descriptors: Morbidity; Women's health; Review.

\section{Resumen}

Objetivo: El estudio objetivó caracterizar los artículos científicos sobre la próxima misión materna. Método: Se trata de una revisión narrativa, del tipo integrativa, con recorte en estudios brasileños. La selección de artículos fue realizada en diciembre de 2016. Resultados: En total se encontraron 33 artículos. Cuando se verificaron los criterios de selección permanecieron siete publicaciones. Los estudios revisados tratan el tema a partir de diferentes perspectivas, con predominio de investigaciones epidemiológicas. Las complicaciones hipertensivas aparecen como el principal causante del fenómeno. Conclusión: Este se trata de un problema de salud pública, que impacta la salud materna y la salud del bebé, sobre el vínculo $y$ en el entorno psicosocial de quien por esta es acometida.

Descriptores: Morbilidad; Salud de la mujer; Revisión.

\section{Introdução}

A trajetória das mulheres em busca por seus direitos advém de décadas atrás. Entre esses direitos encontra-se o acesso aos serviços e ações de saúde de qualidade ${ }^{(1)}$. No entanto, o tema saúde da mulher ainda é foco dos debates e da agenda da saúde pública, e, acima de tudo, desafia-nos a encontrar caminhos e soluções para muitos dos problemas vivenciados. Alguns pontos são antigos e, ao mesmo tempo, inesgotáveis, tendo em vista sua importância, seu impacto, prevalência e 
incidência, sobretudo, problemáticas no âmbito da saúde reprodutiva, como é o caso da morte materna, aborto, gravidez de alto risco, entre outros ${ }^{(2)}$.

Nos últimos anos, o tema mortalidade materna tem sido motivo de preocupação de pesquisadores, sobre tudo, nos países que estão em desenvolvimento e os subdesenvolvidos. Uma vez que, a maior parcela dessas mortes concentrase nos países em desenvolvimento e são decorrentes da falta de acesso a cuidados de rotina adequados $\mathrm{e}$ cuidados de emergência quando necessários ${ }^{(2-3)}$. É importante que se compreenda por que mulheres gestantes adoecem, do que adoecem e quais são as repercussões para sua saúde ${ }^{(2)}$.

A Organização Mundial da Saúde aponta altas estimativas de mulheres que apresentam complicações agudas da gestação, as quais podem estar acompanhadas com a ocorrência de óbitos. O evento da morte materna extrapola a tragédia individual, constituindo um aspecto de avaliação do desenvolvimento humano. No Brasil, o óbito materno é um problema subdimensionado, tendo sido estimado em 2002 pelo DATASUS. São cerca de 73 óbitos maternos por 100 mil nascidos vivos, dado considerado aquém a realidade do fenômeno no país ${ }^{(4)}$.
O Near Miss, também conhecido como morbidade materna grave, caracterizado de acordo com a Organização Mundial da Saúde como uma experiência de quase morte, causado por complicações graves ocorridas com a mulher durante $\mathrm{o}$ período de gravidez, parto ou puerpério, vem ganhando cada vez mais foco, além da mortalidade, por ter sua proporção bem maior do que o evento do óbito materno $^{(2)}$. Sendo importante ainda frisar que, a condição de near miss pode ser um indicador mais útil na análise da assistência obstétrica do que a mortalidade materna em si, a que a própria mulher pode ser uma fonte de $\operatorname{dados}^{(5)}$. A utilização desta problemática, quando aplicado à saúde materna e assistência dispendida a mulher gestante e puérpera, contribui enquanto uma ferramenta importante no combate à morte materna e na melhora da qualidade do cuidado obstétrico oferecido $^{(4)}$.

Souza, Cecatti, Parpinelli, Sousa e Serruya $^{(4)}$ revelam que, ao pensar no índice de mortalidade materna e a razão de near miss por mil partos nos países desenvolvidos, encontra-se, respectivamente, de $3,0 \%$ e 3,4 casos/mil partos, enquanto que nos países em desenvolvimento estes indicadores foram de $7,5 \%$ e 18 
casos/mil partos, o que mostra a maior incidência desse tipo de evento em países que ainda estão se desenvolvendo. É de suma importância ressaltar que a experiência da morbidade materna possui importante impacto sobre a saúde física da mulher, assim como sobre sua saúde emocional.

Vivenciar tal processo, muitas vezes, é ver transformar-se em pesadelo uma fase do ciclo de vida em que mulher está socialmente vinculada ao ideário de felicidade feminina: o exercício da maternidade ${ }^{(6)}$. De acordo com Godoy, Gualda, Bergamasco e Tsunechiro ${ }^{(7)}$, para grande parte da parcela de mulheres que vivenciaram esta experiência, as implicações não foram positivas, já que adquiriram doenças, sofrimento e medos não existentes antes, além de, em alguns casos, dificuldades para concretizar o sonho de ser mãe. Dessa forma, algumas necessitam de acompanhamento psicológico em função das queixas de tristeza, depressão, medo de relacionar-se com as pessoas. É necessário que exista uma compreensão que vá além do estado biológico/patológico. É preciso que a mesma seja olhada como um ser total, buscando o sentido da maternidade, das suas crenças e valores mergulhados em sua cultura ${ }^{(4,7)}$.
Ressalta-se que a maioria dos problemas de saúde da mãe e/ou bebê, que futuramente poderão ser as causas dos óbitos ou quase óbito das mesmas, podem ser detectados nas consultas prénatais. É indispensável, portanto, a assistência de qualidade à gestante durante o pré-natal, bem como, se faz importante uma equipe multidisciplinar capacitada para atender as demandas destas pacientes. Assistência estas que deve ir além das queixas às aflições físicas, contemplando também aspectos emocionais, valores e crenças relacionadas aos problemas apresentados pelas mulheres ${ }^{(8)}$.

Diante do exposto, considerando a relevância deste tema, reforçamos a importância de aprofundar e estudar a experiência de near miss materno, para que se ampliar o olhar sobre $o$ fenômeno em questão. Sendo assim, o presente estudo tem por objetivo caracterizar, por meio de uma revisão integrativa de literatura, os artigos científicos publicados que abordam o tema do near miss materno.

\section{Método}

Este é um estudo de revisão narrativa, do tipo integrativa da literatura. Este método tem sido amplamente utilizado para estudos no 
campo das práticas em saúde, por permitir a formulação de sínteses de investigações que poderão auxiliar na orientação das ações e intervenções por intermédio das evidências cientificas ${ }^{(9)}$. Para o desenvolvimento desta revisão, elaborou-se a seguinte pergunta de pesquisa: "O que abordam os artigos científicos publicados sobre o tema do near miss materno?". As etapas da realização deste estudo foram as seguintes: a) definição da pergunta de revisão; b) critérios de seleção (estratégias de coleta, critérios de inclusão e exclusão); c) realização da coleta; d) elaboração da análise dos estudos selecionados; e) discussão, interpretação e síntese dos resultados; f) apresentação da revisão integrativa.

Para realização das buscas utilizou-se a seguinte expressão: "near miss materno". Estas foram realizadas na base de dados Scientific Eletronic Library Online (SCIELO). Fora também realizada busca eletrônica na Biblioteca Virtual de Saúde (BVS), selecionando publicações disponíveis nas bases de dados Literatura Latino Americana em Ciências da Saúde (LILACS) e Medical Literature Analisys and Retrieval System Online (MEDLINE). Ademais, acrescentou-se material disponível no Portal de Periódicos CAPES, utilizando-se da mesma estratégia de investigação. A seleção de artigos fora realizada em dezembro de 2016, compreendendo publicações disponibilizadas até a realização da busca.

A investigação baseou-se na presença dos termos nos títulos e/ou resumo dos artigos e nas palavras chaves que descreviam o estudo. Foram incluídos nesta revisão: artigos disponíveis na íntegra, que tratassem da temática pretendida e, por fim, estudos brasileiros. Como critério de exclusão estabeleceu-se que seriam eliminados material em formato de livro ou capítulo de livro, dissertação, tese, editorial, comentário, crítica, resenha, anais e erratas. A seleção e aplicação dos critérios de inclusão e exclusão fora realizada por dois juízes. Havendo discordância, um terceiro juiz era consultado para verificar a inclusão ou não do artigo no estudo.

Objetivando caracterizar a produção, organizou-se o material a ser revisado com base nos seguintes critérios: 1 - nome dos autores; 2 - ano da publicação; 3 - periódico de publicação do artigo; 4 - objetivo do estudo; 5 - desenho do estudo. Por fim, apresentaram-se os principais resultados, desfechos e conclusões dos estudos revisados. 


\section{Resultados e Discussão}

Ao todo foram encontrados 33 artigos. Contudo, quando verificados os critérios de inclusão e exclusão, bem como a constatação dos artigos em duplicata, ou seja, disponibilizado em mais de uma base de dados, permaneceu um total de oito publicações, os quais foram escrutinados nessa revisão de literatura. A tabela 1 demonstra uma síntese dos resultados no que concerne aos dados bibliométricos.

Os estudos revisados tratam do tema a partir de diferentes perspectivas e utiliza-se de múltiplas abordagens, com predominância de estratégias epidemiológicas. Um número expressivo das publicações ocorreu em 2015, com um total de três artigos publicados sobre a temática, os quais representam 37,5\% das publicações, seguido de duas publicações respectivamente dos anos de 2013 e 2016 , totalizando $25 \%$ cada, e por fim, um manuscrito publicado no ano de 2014, que representando $12,5 \%$ das publicações A seguir debateremos, brevemente, os dados constantes nos estudos incluídos na revisão.

No que tange as estratégias metodológicas, ressalta-se uma predominância de estudos de caráter quantitativos, epidemiológicos e ecológicos, e apenas um estudo que investiga $\mathrm{o}$ fenômeno na perspectiva qualitativa. Os periódicos que sobressaem nas publicações sobre o escopo em questão são a revista Cadernos de Saúde Pública e Ciência \& Saúde Coletiva, que juntas concentram cinco $(62,5 \%)$ das oito publicações.

Durante a leitura e análise na íntegra, um trabalho fora excluído por tratar-se de um estudo que verificava o desempenho do Sistema de Informações Hospitalares do Sistema Único de Saúde na captação dos casos de near miss materno ocorridos em um serviço de saúde pública. Ou seja, verificou-se, na análise dos juízes, que o foco do trabalho não se tratava do near miss, mas sim da qualidade da informação registrada no SIH-SUS, o que levou a opção de excluir o artigo em questão, permanecendo assim, um total de sete artigos a serem escrutinados integralmente.

Oliveira e $\operatorname{Costa}^{(10)}$, que investigaram a prevalência de óbitos fetais e/ou neonatais em pacientes com near miss materno, verificaram uma prevalência de 19,5\% de óbitos fetais e $7,7 \%$ de mortes neonatais quando há a ocorrência de um quadro de near miss. 
Tabela 1. Dados Bibliométricos dos Artigos Revisados

\begin{tabular}{|c|c|c|c|c|}
\hline $\begin{array}{c}\text { Autoria } \\
\text { (Ano) }\end{array}$ & Periódico & Título & Objetivo & Método \\
\hline $\begin{array}{l}\text { Oliveira } \\
\text { LC, Costa } \\
\text { AAR } \\
(2013)\end{array}$ & $\begin{array}{l}\text { Revista da } \\
\text { Associação } \\
\text { Médica } \\
\text { Brasileira }\end{array}$ & $\begin{array}{l}\text { Óbitos fetais e } \\
\text { neonatais entre } \\
\text { casos de near miss } \\
\text { materno }\end{array}$ & $\begin{array}{l}\text { Caracterizar a } \\
\text { prevalência dos } \\
\text { óbitos fetais e } \\
\text { neonatais entre } \\
\text { pacientes com near } \\
\text { miss materno }\end{array}$ & $\begin{array}{l}\text { Estudo } \\
\text { descritivo, tipo } \\
\text { transversal e } \\
\text { retrospectivo }\end{array}$ \\
\hline $\begin{array}{l}\text { Dias MAB } \\
\text { et al. } \\
(2014)\end{array}$ & $\begin{array}{l}\text { Cadernos de } \\
\text { Saúde } \\
\text { Pública }\end{array}$ & $\begin{array}{l}\text { Incidência do near } \\
\text { miss materno no } \\
\text { parto e pós-parto } \\
\text { hospitalar: dados da } \\
\text { pesquisa Nascer no } \\
\text { Brasil }\end{array}$ & $\begin{array}{l}\text { Estimar a incidência } \\
\text { de near miss materno } \\
\text { no parto e pós-parto } \\
\text { hospitalar }\end{array}$ & $\begin{array}{l}\text { Estudo } \\
\text { epidemiológico }\end{array}$ \\
\hline $\begin{array}{l}\text { Oliveira } \\
\text { LC, Costa } \\
\text { AAR } \\
(2015)\end{array}$ & $\begin{array}{l}\text { Revista } \\
\text { Brasileira de } \\
\text { Terapia } \\
\text { Intensiva }\end{array}$ & $\begin{array}{l}\text { Near miss materno } \\
\text { em unidade de } \\
\text { terapia intensiva: } \\
\text { aspectos clínicos e } \\
\text { epidemiológicos }\end{array}$ & $\begin{array}{l}\text { Avaliar o perfil } \\
\text { clínico } \\
\text { epidemiológico de } \\
\text { mulheres com near } \\
\text { miss materno }\end{array}$ & $\begin{array}{l}\text { Estudo } \\
\text { descritivo, tipo } \\
\text { transversal e } \\
\text { retrospectivo }\end{array}$ \\
\hline $\begin{array}{l}\text { Rosendo } \\
\text { TMSS, } \\
\text { Roncalli } \\
\text { AG } \\
(2015)\end{array}$ & $\begin{array}{l}\text { Ciência \& } \\
\text { Saúde } \\
\text { Coletiva }\end{array}$ & $\begin{array}{l}\text { Prevalência e } \\
\text { fatores associados } \\
\text { ao Near Miss } \\
\text { Materno: inquérito } \\
\text { populacional em } \\
\text { uma capital do } \\
\text { Nordeste Brasileiro }\end{array}$ & $\begin{array}{l}\text { Determinar a } \\
\text { prevalência do near } \\
\text { miss e de } \\
\text { complicações no } \\
\text { período gravídico- } \\
\text { puerperal }\end{array}$ & $\begin{array}{l}\text { Estudo } \\
\text { epidemiológico }\end{array}$ \\
\hline $\begin{array}{l}\text { Souza MA, } \\
\text { Souza TH, } \\
\text { Gonçalves } \\
\text { AK } \\
(2015)\end{array}$ & $\begin{array}{l}\text { Revista } \\
\text { Brasileira de } \\
\text { Ginecologia } \\
\text { e Obstetrícia }\end{array}$ & $\begin{array}{l}\text { Fatores } \\
\text { determinantes do } \\
\text { near miss materno } \\
\text { em uma unidade de } \\
\text { terapia intensiva } \\
\text { obstétrica }\end{array}$ & $\begin{array}{l}\text { Avaliar os fatores } \\
\text { determinantes da } \\
\text { morbimortalidade em } \\
\text { unidade de terapia } \\
\text { intensiva obstétrica }\end{array}$ & $\begin{array}{l}\text { Estudo } \\
\text { observacional de } \\
\text { coorte } \\
\text { transversal }\end{array}$ \\
\hline $\begin{array}{l}\text { Aguiar CA, } \\
\text { Tanaka } \\
\text { ACA } \\
(2016)\end{array}$ & $\begin{array}{l}\text { Cadernos de } \\
\text { Saúde } \\
\text { Pública }\end{array}$ & $\begin{array}{l}\text { Memórias coletivas } \\
\text { de mulheres que } \\
\text { vivenciaram o near } \\
\text { miss materno: } \\
\text { necessidades de } \\
\text { saúde e direitos } \\
\text { humanos }\end{array}$ & $\begin{array}{l}\text { Analisar as memórias } \\
\text { coletivas presentes } \\
\text { em narrativas de } \\
\text { mulheres que } \\
\text { vivenciaram tal } \\
\text { evento }\end{array}$ & $\begin{array}{l}\text { Estudo de } \\
\text { múltiplos casos, } \\
\text { qualitativo, } \\
\text { utilizando a } \\
\text { História Oral }\end{array}$ \\
\hline $\begin{array}{l}\text { Rosendo } \\
\text { TMSS, } \\
\text { Roncalli } \\
\text { AG } \\
(2016)\end{array}$ & $\begin{array}{l}\text { Ciência \& } \\
\text { Saúde } \\
\text { Coletiva }\end{array}$ & $\begin{array}{l}\text { Near miss materno e } \\
\text { iniquidades em } \\
\text { saúde: análise de } \\
\text { determinantes } \\
\text { contextuais no Rio } \\
\text { Grande do Norte, } \\
\text { Brasil }\end{array}$ & $\begin{array}{l}\text { Identificar fatores } \\
\text { socioeconômicos e } \\
\text { de assistência à saúde } \\
\text { na atenção básica } \\
\text { associados ao near } \\
\text { miss materno }\end{array}$ & Estudo ecológico \\
\hline
\end{tabular}

Fonte: elaborado pelos autores do estudo. 
Dentre as mulheres estudadas, predominou o near miss decorrente de distúrbios hipertensivos, como préeclâmpsia grave, e, em seguida, decorrentes da síndrome HELLP, que em inglês significa Hemólise, Enzimas hepáticas elevadas e baixa contagem de plaquetas. Os dados encontrados sugerem elevada a ocorrência de óbitos fetais e neonatais entre as pacientes com near miss materno.

Estes mesmos autores realizaram uma pesquisa que investigou o perfil clínico epidemiológico de mulheres com quadro de near miss. O estudo verificou uma razão de 12,8 casos de near miss para cada 1000 nascidos vivos. O perfil encontrado numa população de 255 casos fora de mulheres com baixo nível de escolarização, em sua maioria primigestas e que tinham realizado cesariana prévia. No que tange ao diagnostico que levou ao near miss, predominou distúrbios hipertensivos, seguido pela síndrome hellp, a qual ocorreu especialmente devido a elevada frequência de plaquetopenia aguda nas mulheres investigadas, a qual também pode ser considerada condição predisponente ao quadro de quase mortalidade ${ }^{(11)}$.

Dias, Domingues, Schilithz, Nakamura-Pereira, Diniz, Brum et al. ${ }^{(3)}$ realizaram um estudo epidemiológico nas 5 regiões brasileiras, que estimou a incidência de near miss materno durante o parto e no pós-parto hospitalar a partir de características das mães. O estudo demonstrou uma incidência de 10,2 casos para cada 1000 nascidos vivos. Neste estudo ainda verificou-se algumas características que associaram-se ao near miss, sendo elas a idade materna acima de 35 anos, histórico de cesariana anterior, gestação de risco e dificuldades de acesso aos serviços de saúde.

Outro estudo realizado em um município do Nordeste brasileiro encontrou uma razão de 41,1 casos para cada 1000 nascidos vivos. Características semelhantes ao estudo anterior foram encontradas neste trabalho, uma vez que se verificaram algumas condições desfavoráveis que sinalizavam para o near miss: idade acima de 35 anos, menor renda, menor escolaridade, maior densidade domiciliar, os quais parecem indicar uma maior vulnerabilidade psicossocial como fator de risco ao near miss ${ }^{(12)}$.

$$
\text { Rosendo e Roncalli }{ }^{(13)}
$$
procederam a uma análise de determinantes contextuais para a ocorrência do fenômeno em um estado nordestino. Nesta análise, os autores verificaram uma razão média de near 
miss materno de 36,7 casos para cada 1000 mulheres. Como condições relacionadas o estudo apontou que a pré-eclampsia, seguida de hemorragia e sepse surgiram como condições marcadoras ou geradoras do quadro. Enquanto determinantes contextuais verificou-se que pobreza e vulnerabilidade psicossocial estão diretamente relacionadas ao quadro. Por outro lado, o estudo averiguou uma correlação negativa significativa com o percentual de famílias com perfil de saúde beneficiário por programas governamentais de transferência de renda.

No estudo realizado por Souza, Souza e Gonçalves ${ }^{(14)}$ encontrou-se algumas características associadas a ocorrência do near miss materno. Tratava-se de mulheres de etnia não branca, casadas, com baixa escolaridade, oriundas de cidades do interior, com baixa renda - renda menor que 1 salário mínimo. Dentre as condições relacionadas ao mesmo estavam a ocorrência ou não de distúrbios hipertensivos gestacionais, realização de pré-natal e via de parto cesárea. $\mathrm{O}$ estudo ressalta a importância do pré-natal, pois este pode auxiliar na identificação de risco, objetivando, por sua vez, diminuir o risco obstétrico e neonatal.

$$
\text { Por fim, Aguiar e Tanaka }{ }^{(6)}
$$

apontaram que as hemorragias foram as principais responsáveis pelo estado de near miss, seguida por complicações hipertensivas. Neste estudo, o único de orientação qualitativa, entrevistou-se doze mulheres, as quais indicaram, em resumo, que as mulheres no estudo não tiveram suas necessidades de saúde atendidas, vivenciaram um conjunto de deficiências assistenciais e violação de direitos, dentre os quais a privação do contato com o filho. O estudo indica que essas mães, que sofrem com o near miss e outras complicações pós-parto encontram conforto em outras vias, que não a jurídica, tais como a militância a favor do parto normal, a busca por profissionais que atuem com parto humanizado em gravidez subsequente e, em alguns casos, formalização de queixa à ouvidoria da maternidade.

\section{Conclusão}

O presente estudo objetivou revisar, na literatura, a produção científica sobre o fenômeno do near miss materno. Este se trata de um problema de saúde pública, uma vez que impacta à saúde materna, bem como a saúde do bebê, sobre o vinculo e laço a ser desenvolvido nesta díade e no entorno psicossocial de quem por este é 
acometido. Complicações hipertensivas parecem ser o principal causador do near miss materno. E a ocorrência deste fenômeno parece sinalizar a falta de uma assistência integral à saúde da gestante, uma vez que tais conjunturas de risco que estão associadas ao near miss, podem ser evitadas. Os achados aqui revisados ressaltam a necessidade de uma assistência integral e humanizada voltada a este público.

$\mathrm{O}$ estudo em cena apresenta algumas limitações, dentre as quais se destaca o recorte para estudos brasileiros, uma vez que seria pertinente revisitar literaturas de outras culturas e países sobre o fenômeno em escrutínio. Verificou-se que a maioria dos estudos esta orientado por uma perspectiva quantitativa e epidemiológica. Nesse sentido, ressalta-se que é imprescindível dar visibilidade a individualidade da história das mulheres que vivenciam o near miss. Logo, sugere-se o desenvolvimento de pesquisas que se debrucem sobre o fenômeno vivenciado pelas mulheres, a partir de uma vertente qualitativa.

\section{Referências}

1. Anjos KF, Santos VC, Souzas R, Eugênio BG. Aborto e saúde pública no Brasil: reflexões sob a perspectiva dos direitos humanos. Saúde debate [periódico na Internet]. 2013 [citado 2017 Jun. 29]; 37(98):504-15. Disponível em: http://dx.doi.org/ 10.1590/S0103-11042013000300014.

2. Godoy SR, Bergamasco RB, Gualda DMR, Tsunechiro MA. Severe obstetric morbidity - near miss. Meaning for surviving women: oral history. Online braz $\mathrm{j}$ nurs [internet newspaper]. 2008 [quoted 2017 Jun. 29]; 7(2):1-1. Available in: http://dx.doi.org/10.5935/1676-4285.2 0081460 .

3. Dias MAB, Domingues RMSM, Schilithz AOC, Nakamura-Pereira M, Diniz CSG, Brum IR et al. Incidência do near miss materno no parto e pósparto hospitalar: dados da pesquisa Nascer no Brasil. Cad. Saúde Pública [periódico na Internet]. 2014 [citado 2017 Jun. 29]; 30(Suppl 1):S169-81. Disponível em: http://dx.doi.org/ 10.1590/0102-311X00154213.

4. Souza JP, Cecatti JG, Parpinelli MA, Sousa MH, Serruya SJ. Revisão sistemática sobre morbidade materna near miss. Cad. Saúde Pública [periódico na Internet]. 2006 [citado 2017 Jun. 29]; 22(2):255-64. Disponível em: http://dx.doi.org/10. 1590/S0102-311X2006000200003.

5. Morse ML, Fonseca SC, Gottgtroy CL, Waldmann CS, Gueller E. Morbidade Materna Grave e Near Misses em Hospital de Referência Regional. Rev. bras. epidemiol. [periódico na Internet]. 2011 [citado 2017 Jun. 29]; 14(2):310-22. Disponível em: http://dx.doi.org/10.15 90/S1415-790X2011000200012. 
6. Aguiar CA, Tanaka ACA. Memórias coletivas de mulheres que vivenciaram o near miss materno: necessidades de saúde e direitos humanos. Cad. Saúde Pública [periódico na Internet]. 2016 [citado 2017 Jun. 29]; 32(9):1-13. Disponível em: http://dx.doi.org/10.15 90/0102-311X00161215.

7. Godoy SR, Gualda DMR, Bergamasco RB, Tsunechiro MA. "Near miss": repercussões e percepção da assistência recebida por mulheres sobreviventes egressas de uma unidade de terapia intensiva. Enfermagem [periódico na Internet]. 2009 [citado 2017 Jun. 29]; 22(2):162-8. Disponível em: http://dx.doi.org/10.1590/S010321002009000200008.

8. Araújo KR, Medeiros TMC, Passos AC, Ribeiro JF. Perfil clínico epidemiológico das pacientes admitidas na unidade de terapia intensiva obstétrica de uma maternidade pública. Gestão \& Saúde [periódico na Internet]. 2015 [citado 2017 Jun. 29]; 6(2):1601-12. Disponível em: :http://dx.doi.org/10. 18673/gs.v6i2.22488

9. Souza MT, Silva MD, Carvalho R. Integrative review: what is it? How to do it?. Einstein (São Paulo) [internet newspaper]. 2010 [quoted 2017 Jun. 29]; 8(1):102-6. Available in: http://dx.doi.org/10.1590/s1679-45082 010 rw1134.

10. Oliveira LC, Costa AAR. Óbitos fetais e neonatais entre casos de near miss materno. Rev. Assoc. Med. Bras. [periódico na Internet]. 2013 [citado 2017 Jun. 29]; 59(5):487-94. Disponível em: http://dx.doi.org/10.10 16/j.ramb.2013.08.004.
11. Oliveira LC, Costa AAR. Near miss materno em unidade de terapia intensiva: aspectos clínicos e epidemiológicos. Rev. bras. ter. intensiva [periódico na Internet]. 2015 [citado 2017 Jun. 29]; 27(3):220-7. Disponível em: http://dx.doi.org/10.59 35/0103-507X.20150033.

12. Rosendo TMSS, Roncalli AG. Prevalência e fatores associados ao Near Miss Materno: inquérito populacional em uma capital do Nordeste Brasileiro. Ciênc. saúde coletiva [periódico na Internet]. 2015 [citado 2017 Jun. 29]; 20(4):1295-304. Disponível em: http://dx.doi.org/10.15 90/1413-81232015204.09052014.

13. Rosendo TMSS, Roncalli AG. Near miss materno e iniquidades em saúde: análise de determinantes contextuais no Rio Grande do Norte, Brasil. Ciênc. saúde coletiva [periódico na Internet]. 2016 [citado 2017 Jun. 29]; 21(1):191-201. Disponível em: http://dx.doi.org/10.1590/1413-812320 15211.20802014.

14. Souza MAC, Souza THSC, Gonçalves AKS Fatores determinantes do near miss materno em uma unidade de terapia intensiva obstétrica. Rev. Bras. Ginecol. Obstet. [periódico na Internet]. 2015 [citado 2017 Jun. 29]; 37(11):498-504. Disponível em: http://dx.doi.org/10.1590/SO100-7203 20150005286. 